J. Asiat. Soc. Bangladesh, Sci. 40(2): 249-258, December 2014

\title{
A STUDY OF THE ALPHA - NUCLEUS SCATTERING
}

\author{
D. R. SARKER ${ }^{\prime}$, AIN-UL-HUDA' ${ }^{\prime}$, S. K. DAS ${ }^{\prime}$, MD. K. HASAN' ${ }^{\prime}$ AND MD. M. PARVEJ' \\ MD. A. RAHMAN ${ }^{2}$ AND H. M. SEN GUPTA ${ }^{2}$ \\ 'Department of Physics, Jagannath University, Dhaka, Bangladesh \\ 2 Department of Physics. University of Dhaka, Bangladesh
}

\begin{abstract}
Angular distribution data for the elastic scattering of $1.37 \mathrm{GeV}$ alpha particles from several nuclei are analyzed in terms of the three parameter strong absorption model of Frahn and Venter. The fits are quite satisfactory over practically the entire angular range and the best fit parameters are obtained. These are used for the study of the inelastic scattering of alpha particles leading to the collective states in nuclei. A reasonably good fit is obtained without any adjustment of the parameters suggesting thereby the success of the strong absorption model. Deformation parameters are extracted for the collective states in nuclei.
\end{abstract}

Key words: Nuclear reactions and scattering: $(\alpha, \alpha),\left(\alpha, \alpha^{\prime}\right)$, Optical and diffraction models, Elastic ${ }^{3} \mathrm{He}$ scattering, Inelastic ${ }^{3} \mathrm{He}$ scattering

\section{Introduction}

Interaction between nucleons and nuclear particles with nucleus is a complicated manybody problem and it is extremely difficult, if not impossible, to handle the interaction process from theoretical considerations. Different approximations, i.e. different models have proposed to describe the interaction. The most commonly used one is then optical model for the elastic process and there from the Distorted wave Born approximation (DWBA) and the coupled channel Born approximation (CCBA) to study various nonelastic processes (inelastic scattering of the incident particles leading to collective states in nuclei and different nuclear reactions).

The process of the many - body interactions is replaced in the optical model by an average two - body potential. The model has been found to be quite successful in describing the interaction of various incident particles over a wide range of energy with various nuclei. However, except under special circumstances, the model suffers from ambiguities in the parameter values. An alternative approach is to represent the nuclear interaction as a diffraction process. Here the target nucleus is considered, as if, to be a 'black' sphere or nearly so such that the incident 'waves' representing the incoming particles, with angular moment less than certain critical or cut-off values are totally absorbed and those larger than the critical value suffer no interaction at all. The critical value of the angular momentum as above depends on the nature of the incident particle, its energy, as well as the target nucleus. A number of diffraction models has been proposed from time to time. We use here the one due to Frahn and his co-workers (Frahn et al. 1963, Frahn 1972, Frahn 1976 and Frahn et al. 1978) which is a generalization of 
others and is termed also as the strong absorption model (SAM). The model is expected to be useful for the interaction of alpha particles with nuclei. Various experimental data are taken from (Alkhazov 1977 and Satchler 1974). The other part of the work is concerned with the studies of inelastic scattering of alpha particles using the SAM parameters obtained from the elastic scattering. It has been emphasized by Scatchler that the real test of the parameters as obtained from the elastic scattering lies to the extent of their ability to account for the non-elastic process.

\section{Materials and Methods}

The strong absorption model: The strong absorption model (Frahn et al. 1963, Frahn 1972, Frahn 1976 and Frahn et al. 1978) starts with a partial wave expansion for the amplitude for elastic scattering of nucleons and nuclear particles from nuclei in terms of the scattering function $\mathrm{n}_{1}$ as given by

$$
\eta_{l \exp (-2 \mathrm{i}} \sigma_{l)=\mathrm{g}(\lambda)+\mathrm{i} \mu} \frac{\operatorname{dg}(\lambda)}{\lambda} \ldots \ldots \ldots
$$

Where $\sigma_{l}$ is the Coulomb phase shift for the $l$ the partial wave and $g(\lambda)$ is a continuous monotonic function of the angular momentum. There is no restriction on the choice of except that it is a continuously differentiable function of the angular momentum $\lambda$ and that its first derivative has a simple Fourier transform. A convenient form of $g(\lambda)$ is the woods - Saxon form, namely,

$$
g(\lambda)=[1+\exp \{(\Lambda-\lambda) / \Delta\}]^{-1} \ldots \ldots \ldots
$$

The function $g(\lambda)$ is thus characterized by the cut - off (i.e. the critical ) angular momentum $\wedge$ that corresponds to the grazing incidence of the incoming partial wave on the nucleus surface, the rounding parameter $\Delta$ and the nuclear phase shift (more accurately $\mu / 4 \dot{\Delta}$ ).

A closed form expression for the elastic cross section is then arrived at (Frahn et al. 1963 and Frahn 1976) in terms of three adjustable parameters, namely $\Lambda, \Delta$ and $\mu$. Parameters $\Lambda$ and $\Delta$ are related respectively to the interaction radius $R$ and the surface diffuseness $d$ through the semi - classical expressions given by

$$
\begin{gathered}
\Lambda=k R\left(1-\frac{2 n}{k R}\right)^{1 / 2} \\
\text { and } \\
\Delta=k d\left(1-\frac{n}{k R}\right)\left(1-\frac{2 n}{k R}\right)^{-1 / 2}
\end{gathered}
$$

where $\mathrm{n}$ and $\mathrm{k}$ are respectively the Coulomb parameter and the wave number. 
It can be seen from relations (1) and (2) that the real part of $\eta_{l} \exp \left(-2 \mathrm{i} \sigma_{l}\right)$ changes from small values at low $l$ to unity at high $l$ through a rapid transition around the critical value, which the imaginary part is clearly surface peaked, being commensurate with the strong absorption situation.

The formalism for elastic scattering as above can be readily extended to include inelastic scattering leading to collective states in nuclei (Austern 1965, Potgieter 1966 and Rahman 1992). The inelastic scattering amplitude of multipole $\mathrm{L}$ can be expressed in terms of the first derivative of the scattering matrix $\eta_{l}$ used to describe the elastic Scattering process. Closed expressions are obtained for the differential cross section for inelastic scattering. The parameters (namely $T, \Delta$ and $\mu$ ) are given from the elastic scattering. The only free parameter is the deformation length $\delta_{1}\left(=\beta_{L} R\right)$, with $\beta_{L}$ as the deformation parameter for the $\mathrm{L}$ th mode of excitation of the nucleus and this is obtained from the normalization of the theoretical cross section to the experimental cross section. Explicit expressions for the differential scattering cross sections for the inelastic scattering cross sections are given by Potgieter 1966 and Rahman 1966.

Finally the total reaction cross section $\sigma_{R}$ is calculated using the following expression, as given by Frahn and Venter

$$
\sigma_{R}=\frac{\pi \wedge^{2}}{k^{2}}\left[1+\frac{2 \Delta}{\wedge}+\frac{1}{3} \pi^{2}\left(\frac{\Delta}{\wedge}\right)^{2}-\frac{1}{3}\left(\frac{\mu}{\Delta}\right)^{2}\left(\frac{\Delta}{\lambda}\right)\right] \ldots \ldots \ldots \ldots
$$

Analyses of data: The parameter $\wedge$ determines the frequency of the oscillation in the angular distribution of elastic scattering. An increase in $\wedge$ shifts the positions of the maxima and minima, hence the whole diffraction pattern laterally towards the smaller angles. The parameter $\Delta$ controls the backward to the forward scattering through which the average slope of the angular distribution is fixed. The higher angle regions are mainly affected by a variation of $\Delta$; an increase is $\Delta$ mainly lowers the maxima of the angular distribution keeping the oscillatory pattern practically unaltered. A variation of $\mu$ affects the cross sections at the minima with an average slope decreasing; the effect on the cross sections at the minima is the most significant. To start with an analysis of the angular distribution data, the parameters $\Delta$ and $\mu$ were given reasonable values and the parameter $\wedge$ was varied to get as close as possible the positions of the maxima and minima in the angular distribution. Having obtained a working value of $\Lambda$ the other two parameters, namely $\Delta$ and $\mu$, were adjusted. Finally, all three parameters were adjusted to arrive at the value of the usual $\chi 2$ minimum.

No adjustment of any of the parameters values was made for studying the angular distributions of the inelastic scattering of alpha particles. 


\section{Results and Discussion}

Elastic Scattering: Results of the least squares SAM analyses of the angular distributions for elastic scattering are summarized in table 1 and the fits are shown in Figs. 1 and 2. Parameters of the model are uniquely given form these analyses and no combination of the parameter values other than the ones summarized in Table 1 could be found that gave another minimum is the $\chi^{2}$ value. This unique can be understood from the fact that the two most important parameters of the SAM, namely the cut-off angular momentum $A$ and the rounding parameters $\Delta$ are related respectively to the interaction radius $R$ and the Surface diffuseness d' through the well-known expressions given by relations 3 and 4 . Uncertainties in the values of $\Lambda$ and $\Delta$ are about $5 \%$ and of $\mu$ are about $20 \%$. The latter is not a very sensitive parameter either; it affects the cross sections at the minima of angular distributions, where the measured values are usually less accurate than at other angles.

The overall fits to the measured angular distributions for elastic scattering are reasonably good and are of similar nature as achieved with the optical model. The latter is characterized by six parameter (sometimes more), as against which the SAM as three. Fits to the lower energy data on light nuclei for both SAM and the optical model are not that satisfactory throughout the entire angular range (Dipika 2010).

The cut- off angular momentum $\wedge$ as expected, increases smoothly with the target mass number as well as with an increase in energy of the incident particles. These certainly speak of the reliability of the parameter. The rounding parameter $\Delta$, with a few exception, also shows a similar behavior as $\Lambda$. The value of the phase shift $\mu / 4 \Delta$, however, shows no systematic variation either with the target mass or with the incident energy.

We now look into the systematics of the interaction radius $R$, the surface diffuseness $d$ and the total reaction cross section $\sigma_{R}$, as obtained from the best- fit SAM parameters. These are shown in Table 1, as given by relations (3-5).

Table 1. SAM parameters for the alpha particles.

\begin{tabular}{|c|c|c|c|c|c|c|c|c|c|}
\hline \multirow{2}{*}{$\begin{array}{l}\text { Target } \\
\text { Nucleus }\end{array}$} & \multirow{2}{*}{$\begin{array}{c}E_{a} \\
(\mathrm{GeV})\end{array}$} & \multicolumn{4}{|c|}{ SAM Parameters } & \multirow{2}{*}{$\chi^{2}$} & \multicolumn{3}{|c|}{ Derived quantities } \\
\hline & & $\wedge$ & $\Delta$ & $\mu$ & $\mu / 4 \Delta$ & & $\begin{array}{c}R \\
(\mathrm{f} m)\end{array}$ & $\begin{array}{c}d \\
(f \mathrm{~m})\end{array}$ & $\begin{array}{c}\sigma_{\mathrm{R}} \\
(\mathrm{mb})\end{array}$ \\
\hline${ }^{12} \mathrm{C}$ & 1.37 & 38.00 & 6.8 & 0.1 & 0.004 & 20.1 & 3.13 & 0.55 & 450.3 \\
\hline${ }^{40} \mathrm{Ca}$ & 1.37 & 74.02 & 10.0 & 1.6 & 0.040 & 9.8 & 4.70 & 0.63 & 912.7 \\
\hline${ }^{42} \mathrm{Ca}$ & 1.37 & 75.50 & 10.5 & 1.4 & 0.034 & 10.0 & 4.87 & 0.67 & 990.7 \\
\hline${ }^{44} \mathrm{Ca}$ & 1.37 & 78.00 & 10.8 & 1.4 & 0.032 & 12.0 & 5.00 & 0.69 & 1045.3 \\
\hline${ }^{48} \mathrm{Ca}$ & 1.37 & 79.50 & 10.4 & 1.2 & 0.029 & 21.0 & 5.07 & 0.66 & 1056.3 \\
\hline${ }^{58} \mathrm{Ni}$ & 1.37 & 86.00 & 10.0 & 3.6 & 0.090 & 26.1 & 5.40 & 0.62 & 1153.8 \\
\hline
\end{tabular}


It is evident from our studies that the value $\mathrm{R}$ increases as the target mass increase, for the same energy as expected (Fig.3). The surface diffuseness $d$ is approximately the constant. Fig. 4 shows a linear relation between $\mathrm{d}$ and $A^{1 / 3}$.

$$
d=0.07 \mathrm{~A}^{1 / 3}+0.43
$$

The mass number dependence of the total reaction cross section $\sigma_{R}$ was next studied. The value of $\sigma_{R}$ increases almost smoothly as the target mass increase when the beam energy remains the same as expressed by the least squares relation

$$
\sigma_{R}=45.04 A^{1 / 3}-58.28(\mathrm{mb})
$$
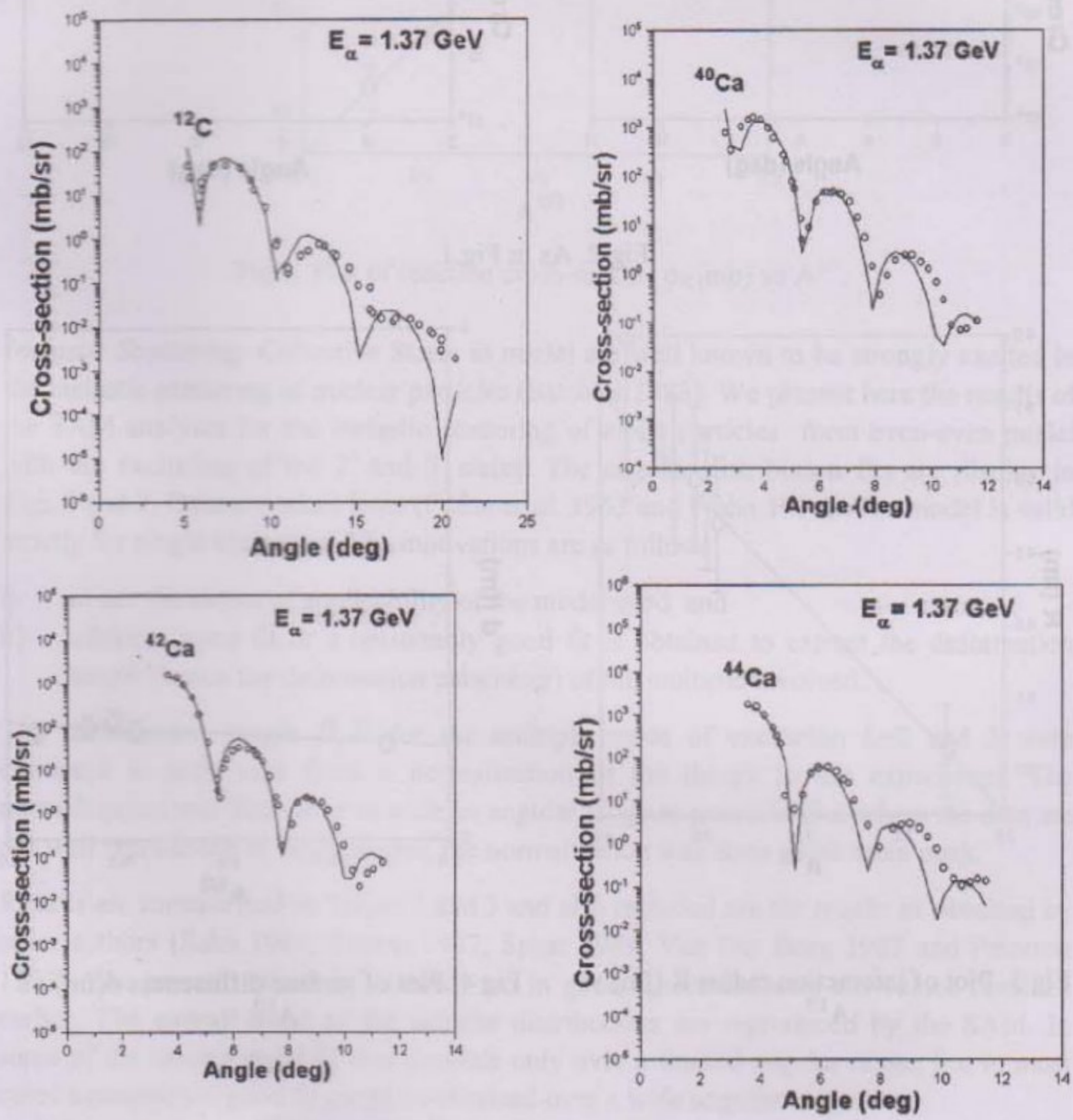

Fig 1. SAM fit of the elastic scattering of alpha particles from different nuclei. 

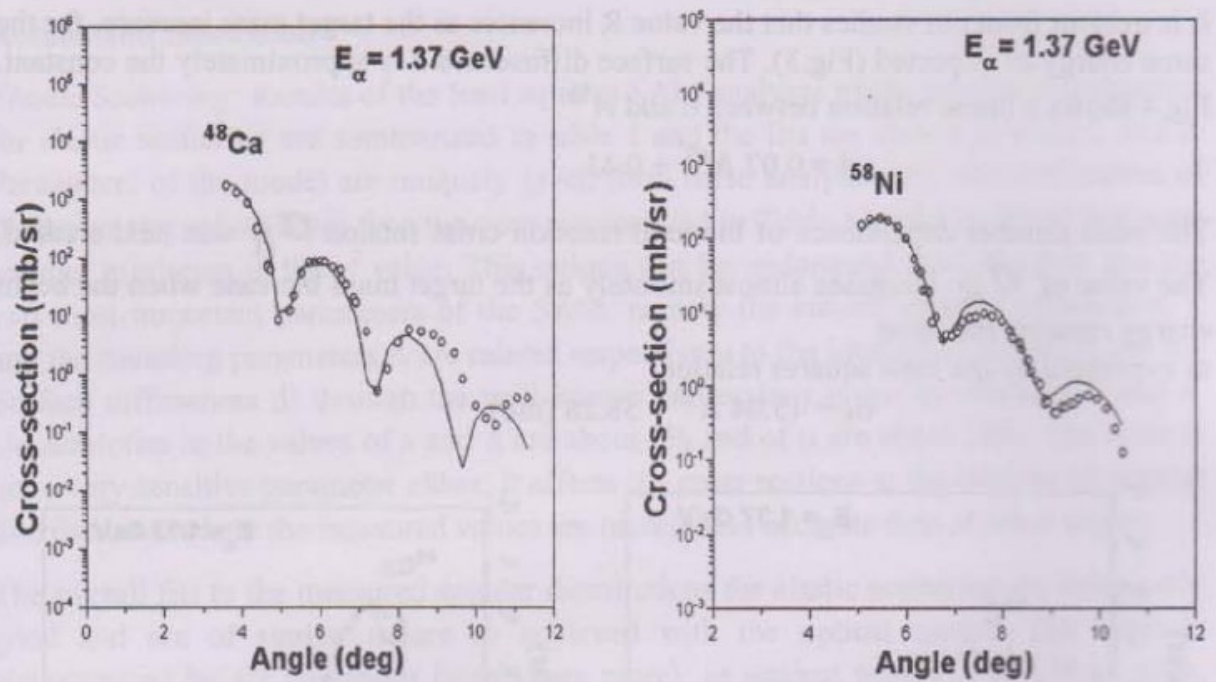

Fig 2. As in Fig.1
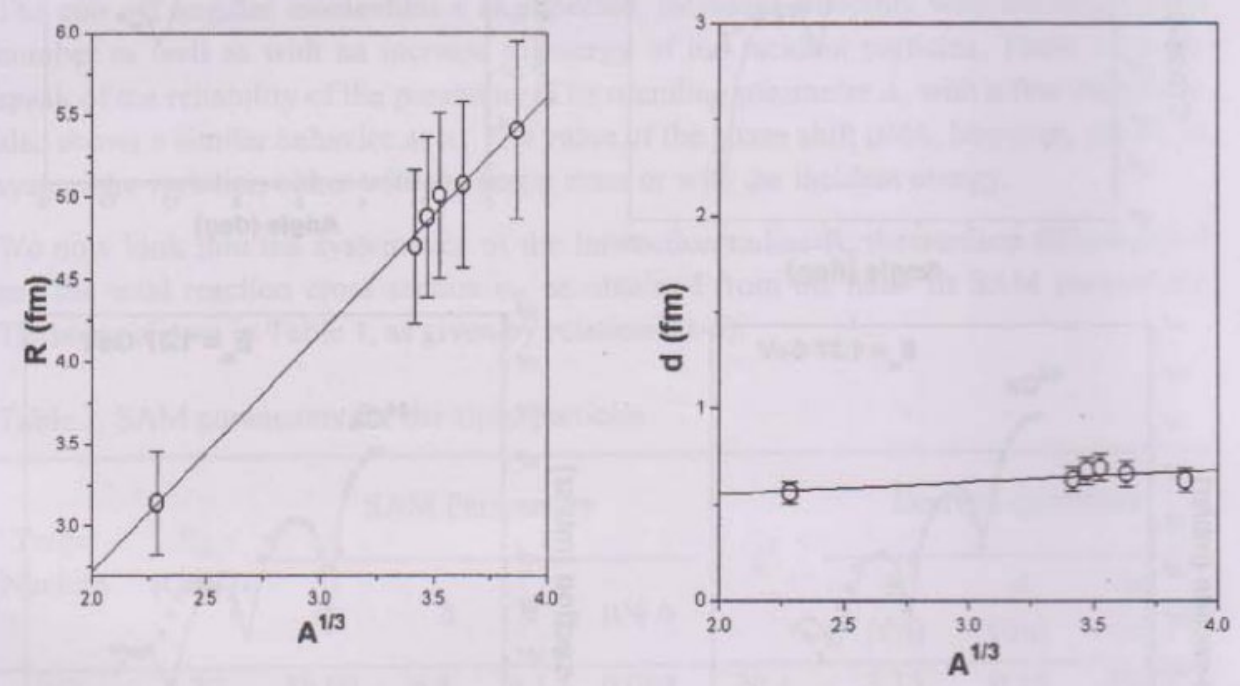

Fig 3. Plot of interaction radius $\mathrm{R}$ ( $\mathrm{fm}$ ) vs $A^{1 / 3}$.

Fig 4. Plot of surface diffuseness $d(f m)$ vs $A^{1 / 3}$. 


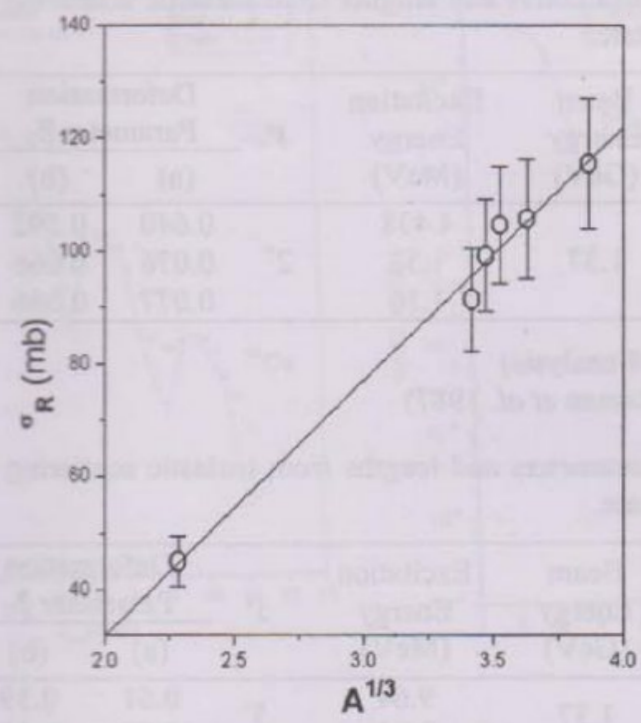

Fig 5. Plot of reaction cross-section $\sigma_{\mathrm{R}}(\mathrm{mb})$ vs $\mathrm{A}^{1 / 3}$.

Inelastic Scattering: Collective States in nuclei are well known to be strongly excited in the inelastic scattering of nuclear particles (Satchler 1983). We present here the results of the SAM analyses for the inelastic scattering of alpha particles form even-even nuclei with the excitation of the $2^{+}$and $3^{*}$ states. The angular distribution fits are display in Figs. 6 and 7. Data are taken from (Frahn et al. 1963 and Frahn 1976). The model is valid strictly for single excitation. The motivations are as follows:

i) to see the extent of applicability of the model used and

ii) where a good fit or a reasonably good fit is obtained to extract the deformation length (hence the deformation parameter) of the multiple involved.

The deformation length $\beta_{L} R$ for the multiple mode of excitation $\mathrm{L}=2$ and 3 were extracted in each case from a normalization of the theory to the experiment. The normalization was done over as wide an angular range as possible. But where the data are not well reproduced at larger angles, the normalization was done at the main peak.

Results are summarized in Tables 2 and 3 and also included are the results as obtained by other authors (Saha 1984, Raman 1987, Spear 1989, Van Der Borg 1987 and Peterson 1993). The present values in all cases are in good agreement with the values obtained earlier. The overall trend of the angular distributions are reproduced by the SAM. In some of the cases a good fit was possible only over a limited angular range, but in most cases a reasonably good fit could be obtained over a wide angular range. 
Table 2. Deformation parameters and lengths from inelastic scattering of $\alpha$ particles for the lowest $2^{+}$state.

\begin{tabular}{|c|c|c|c|c|c|c|c|}
\hline \multirow{2}{*}{$\begin{array}{l}\text { Serial } \\
\text { No. }\end{array}$} & \multirow{2}{*}{$\begin{array}{c}\text { Target } \\
\text { Nucleus }\end{array}$} & \multirow{2}{*}{$\begin{array}{l}\text { Beam } \\
\text { Energy } \\
(\mathrm{GeV})\end{array}$} & \multirow{2}{*}{$\begin{array}{c}\text { Excitation } \\
\text { Energy } \\
(\mathrm{MeV})\end{array}$} & \multirow[t]{2}{*}{$\mathrm{J}^{\pi}$} & \multicolumn{2}{|c|}{$\begin{array}{l}\text { Deformation } \\
\text { Parameter } \beta_{2}\end{array}$} & \multirow{2}{*}{$\begin{array}{l}\text { Deformation } \\
\text { Length } \\
\left(\beta_{2} R\right)(f \mathrm{~m})\end{array}$} \\
\hline & & & & & (a) & (b) & \\
\hline 1 & ${ }^{12} \mathrm{C}$ & & 4.438 & & 0.640 & 0.592 & 1.98 \\
\hline 2 & $42 \mathrm{CA}_{4}$ & 1.37 & 1.52 & $2^{+}$ & 0.076 & 0.066 & 0.39 \\
\hline 3 & ${ }^{44} \mathrm{Ca}$ & & 1.16 & & 0.077 & 0.066 & 0.40 \\
\hline
\end{tabular}

a. Present Work (SAM analysis)

b. Previous Works, (Raman et al. 1987)

Table 3. Deformation parameters and lengths from inelastic scattering of $\alpha$ particles for the lowest 3 ' state.

\begin{tabular}{|c|c|c|c|c|c|c|c|}
\hline \multirow{2}{*}{$\begin{array}{c}\text { Serial } \\
\text { No. }\end{array}$} & \multirow{2}{*}{$\begin{array}{c}\text { Target } \\
\text { Nucleus }\end{array}$} & \multirow{2}{*}{$\begin{array}{l}\text { Beam } \\
\text { Energy } \\
(\mathrm{GeV})\end{array}$} & \multirow{2}{*}{$\begin{array}{c}\text { Excitation } \\
\text { Energy } \\
(\mathrm{MeV})\end{array}$} & \multirow[t]{2}{*}{$\mathrm{J}^{\pi}$} & \multicolumn{2}{|c|}{$\begin{array}{l}\text { Deformation } \\
\text { Parameter } \beta_{2}\end{array}$} & \multirow{2}{*}{$\begin{array}{l}\text { Deformation } \\
\text { Length } \\
\left(\beta_{2} R\right)(f m)\end{array}$} \\
\hline & & & & & (a) & (b) & \\
\hline $\begin{array}{l}1 \\
2\end{array}$ & $\begin{array}{l}{ }^{12} \mathrm{C} \\
{ }^{40} \mathrm{Ca}\end{array}$ & 1.37 & $\begin{array}{l}9.64 \\
3.73\end{array}$ & $3^{*}$ & $\begin{array}{l}0.61 \\
0.35\end{array}$ & $\begin{array}{l}0.59 \\
0.34\end{array}$ & $\begin{array}{l}1.64 \\
1.90\end{array}$ \\
\hline
\end{tabular}

a. Present Work (SAM analysis)

b. Previous Works, (Spear 1989)

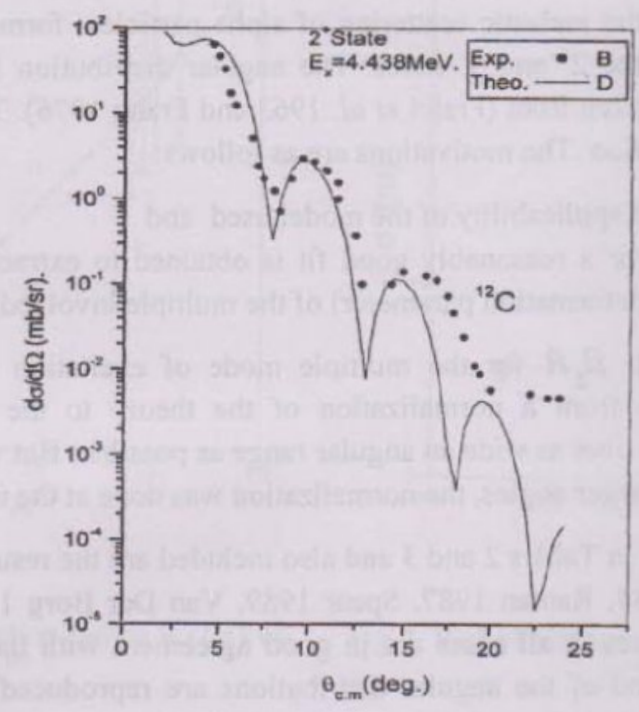



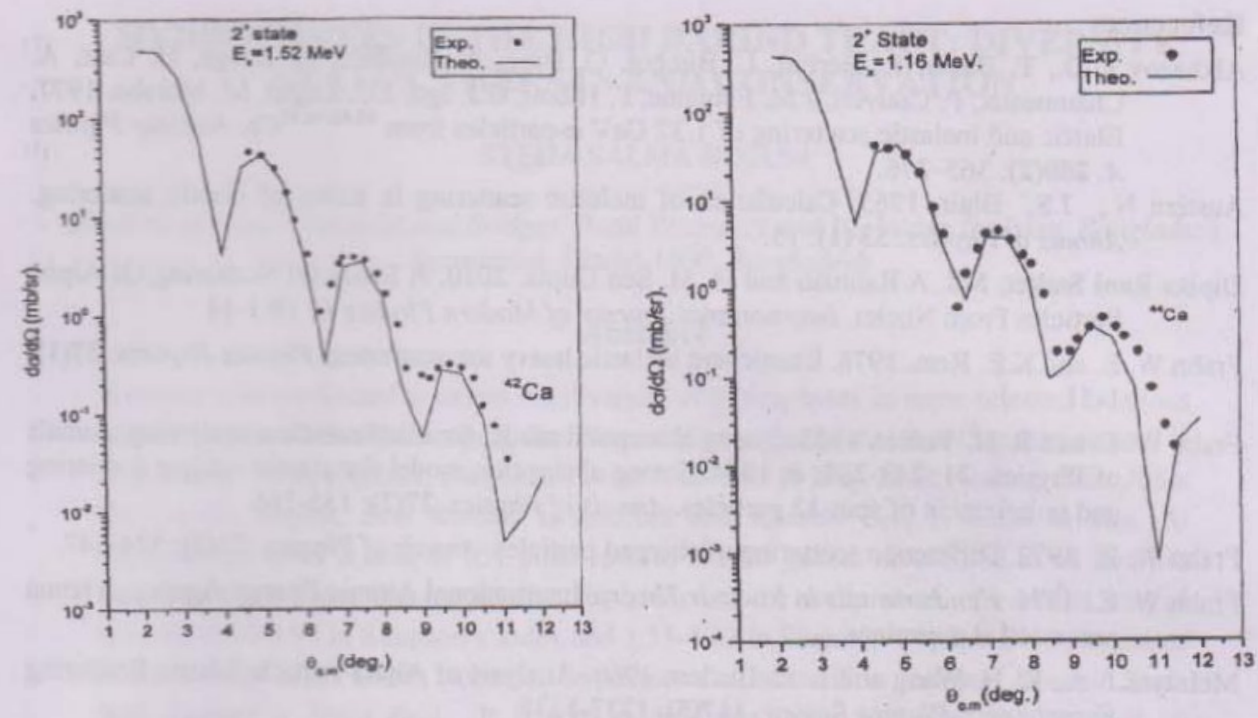

Fig 6. SAM analysis of inelastic scattering of $\alpha$ particles from ${ }^{12} \mathrm{C}$ and ${ }^{42,}{ }^{44} \mathrm{Ca}$ at 1.37 $\mathrm{GeV}$.
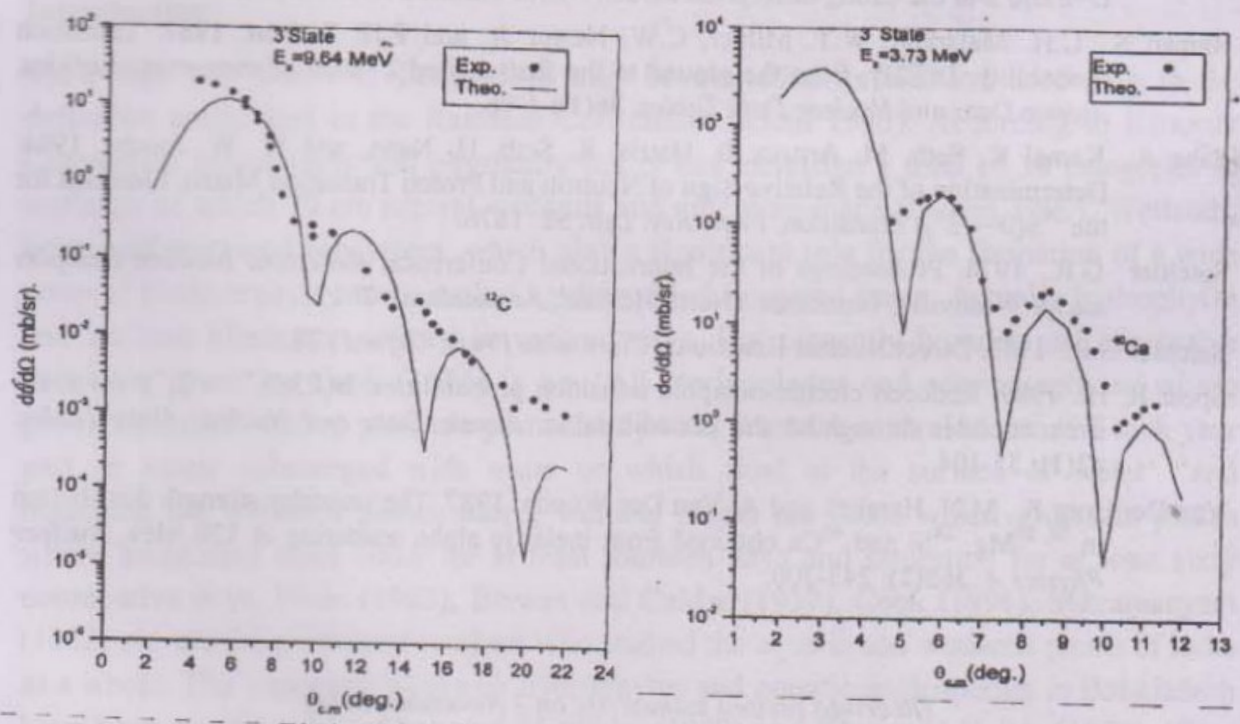

Fig 7. SAM analysis of inelastic scattering of $\alpha$ particles from ${ }^{12} \mathrm{C}$ and ${ }^{40} \mathrm{Ca}$ at $1.37 \mathrm{GeV}$. 


\section{References}

Alkhazov G.D., T. Bauer, R. Bertini, L. Bimbot, O. Bing, A. Boudard, G. Bruge, H. Catz, A. Chaumeaux, P. Couvert, J.M. Fontaine, F. Hibou, G.J. Igo, J.C. Lugol, M. Matoba. 1977. Elastic and inelastic scattering of $1.37 \mathrm{GeV} \alpha$-particles from ${ }^{40,42,44,48} \mathrm{Ca}$. Nuclear Physics A. 280(2): 365-376.

Austern N., J.S. Blair, 1965. Calculation of inelastic scattering in terms of elastic scattering. Annals of Physics. 33 (1): 15.

Dipika Rani Sarker, Md. A Rahman and H. M. Sen Gupta. 2010. A Study Of Scattering Of Alpha Particles From Nuclei. International Journal of Modern Physics E. 19:1-14.

Frahn W. E. and K.E. Rem. 1978. Elastic and inelastic heavy ion scattering. Physics Reports. 37(1): $1-51$.

Frahn W. E. and R. H. Venter. 1963. Strong absorption model for elastic nuclear scattering. Annals of Physics. 24: 243-288. \& 1964. Strong absorption model for elastic nuclear scattering and polarization of spin-12 particles. Annals of Physics. 27(2): 135-166.

Frahn W. E.. 1972. Diffraction scattering of charged particles. Annals of Physics. 72(2): 524-547.

Frahn W. E.. 1976. Fundumentals in Nuclear Theory. International Atomic Energy Agency, Vienna presented at a seminar.

McIntyre J. A., K. H. Wang and L. C. Becker. 1960. Analysis of Alpha-Particle Elastic Scattering Experiments. Physics Review. 117(5): 1337-1338.

Peterson R. J.. 1993. Isospin symmetry in nuclear transitions from pion scattering. Physics Review C. 48: 1128 .

Potgieter J.M. and W.E. Frahn. 1966. Strong absorption model for inelastic nuclear scattering. Nuclear Physics. 80(2): 434-448.

Rahman Md. A., H. M. Sen Gupta. 1992. Nuclear inelastic-scattering cross-section for transitions $\mathrm{L}=2$ and 3 in the strong-absorption model. Nuovo Cimento A. 105(5): 729-734.

Raman S., C.H. Malarkey, W.T. Milner, C.W. Nestor Jr. and P.H. Stelson. 1987. Transition probability, B(E2) $\uparrow$, from the ground to the first-excited $2^{+}$state of even-even nuclides. Atomic Data and Nuclear Data Tables. 36(1): 1-96.

Saha A., Kamal K. Seth, M. Artuso, B. Harris, R. Seth, H. Nann, and W. W. Jacobs. 1984. Determination of the Relative Sign of Neutron and Proton Transition Matrix Elements for the ${ }^{34} \mathrm{~S}\left(0 \rightarrow 2^{+}\right)$Transition. Phys. Rev. Lett. 52: 1876.

Satchler G.R. 1974. Proceedings of the International Conference, Reactions between Complex nuclei. Nashville, Tennessee. (North Holland, Amsterdam). 2: 171.

Satchler G.R.. 1983. Direct Nuclear Reactions. Clarendon Press, Oxford : 78.

Spear R. H. 1989. Reduced electric-octupole transition probabilities, $\mathrm{B}\left(\mathrm{E} 3 ; 0_{1}{ }^{+} \rightarrow 3_{1}\right)$, for eveneven nuclides throughout the periodic table. Atomic Data and Nuclear Data Tables. 42(1): 55-104.

Van Der Borg K., M.N. Harakeh and A. Van Der Woude. 1987. The isoscalar strength distribution in ${ }^{24,}{ }^{26} \mathrm{Mg}$, ${ }^{28} \mathrm{Si}$ and ${ }^{40} \mathrm{Ca}$ obtained from inelastic alpha scattering at $120 \mathrm{Mev}$. Nuclear Physics A. 365(2): 243-300.

(Received revised manuscript on 7 November 2014) 